\title{
Contrast echocardiography during pericardiocentesis
}

\author{
T R Betts, J R Radvan
}

A 35 year old man presented to the casualty department with a two week history of malaise, dyspnoea, and pleuritic chest pain. His temperature was $38^{\circ} \mathrm{C}$, blood pressure $100 /$ $75 \mathrm{~mm} \mathrm{Hg}$, pulse 105 beats/min and regular, and the heart sounds were quiet. A 12 lead ECG was unremarkable. Chest radiography showed an enlarged cardiac silhouette. Transthoracic echocardiography confirmed a $2 \mathrm{~cm}$ pericardial effusion. Pericardiocentesis was performed via the subxiphoid route with the aid of contrast echocardiography (fig 1). Serology and other investigations suggested a viral aetiology. The catheter was removed after 48 hours and the patient successfully discharged home.

\section{Discussion}

When aspirating pericardial fluid it is important to be sure that the needle tip is in the pericardial space and not a ventricle or pleural cavity. Difficulties may arise as perforation into a ventricle does not always produce ventricular ectopic beats or cause chest pain. Additionally, pericardial fluid is often haemorrhagic and may be pulsatile. Echocardiographic or fluoroscopic guidance is recommended. Most texts describing pericardiocentesis do not detail how echocardiography is best used to ensure a safe procedure. It is implied that imaging is used to visualise the tip of the needle in the pericardial space. In our experience this is often difficult. Contrast echocardiography allows accurate assessment of needle tip position.

The needle should be advanced by the most appropriate route (usually subxiphoid). When fluid is withdrawn, $5 \mathrm{ml}$ of contrast solution is

Cardiology Specialist Registrar, Wessex Cardiothoracic Centre, Mailpoint 46, Southampton General Hospital, Tremona Road, Southampton SO16 6YD, UK

T R Betts

Royal Bournemouth Hospital, Castle Lane East, Bournemouth BH7 7DW, UK

J R Radvan

Correspondence to: Dr Betts.

Accepted for publication 10 August 1998

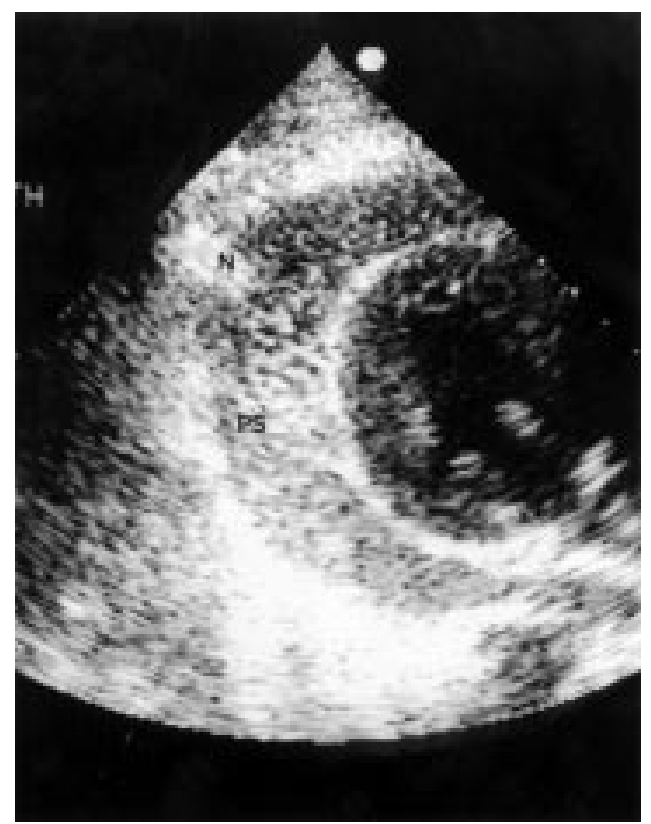

Figure 1 Cross sectional contrast echocardiography, parasternal short axis view, showing pericardial space (PS) containing a cloud of echogenic microbubbles. (L, left ventricle; $N$, needle.)

the precise position of the needle tip in all 16 patients (including the left and right ventricle). When fluid was difficult to aspirate the technique also demonstrated when the needle was in the correct space but against the wall of the ventricle; manipulation of the needle allowed successful aspiration. Other authors have recommended placement of a drain before injecting contrast to avoid laceration of the right ventricle by the needle tip. ${ }^{2}$ When the drain was found to be in a ventricle surgical removal was advised. We believe that contrast can be injected through the needle with no extra risk to the patient, particularly with large effusions.

This case report is a reminder that contrast echocardiography is a simple, effective technique that aids pericardiocentesis. We suggest it should be employed routinely, especially when fluoroscopy is not available.

\footnotetext{
1 Chandrarantna PAN, Reid CL, Nimalasuriya A, et al. Application of 2-dimensional contrast studies during pericardiocentesis. Am f Cardiol 1983;52:1120-2.

2 Weisse AB, Desai RR, Rajihah G, et al. Contrast echocardiography as an adjunct in haemorrhagic or complicated pericardiocentesis. Am Heart f 1996;131:8225 .
}

doctive technique to locate positic directly visualised in nine of 16 patients but the use of contrast allowed the operator to locate 